\title{
Effects of Phase Separation on the Crystallization Behavior in a Binary Blend of Poly( $\varepsilon$-caprolactone) Homopolymer and Poly( $\varepsilon$-caprolactone)-block-Polybutadiene Copolymer
}

\author{
Michiaki AKABA and Shuichi NOJIMA ${ }^{\dagger}$ \\ Department of Polymer Chemistry, Tokyo Institute of Technology, \\ H-125, 2-12-1 Ookayama, Meguro-ku, Tokyo 152-8552, Japan \\ (Received February 10, 2005; Accepted May 11, 2005; Published August 15, 2005)
}

\begin{abstract}
The crystallization process of poly( $\varepsilon$-caprolactone) (PCL) chains in a binary crystalline/crystalline blend, PCL homopolymer and PCL-block-polybutadiene diblock copolymer (PCL- $b$-PB), has been investigated by synchrotron small-angle X-ray scattering (SR-SAXS) as a function of the characteristic length of composition variation $\xi$ formed by the phase separation between PCL and PCL- $b$-PB. The temperature range of an UCST-type phase separation overlaps with that of crystallization in this blend, so that the phase separation starts in advance to yield various heterogeneous structures when the blend is quenched from a microphase-separated melt into the crystallization temperature of PCL chains. When $\xi<500 \mathrm{~nm}$ the crystallization rate of PCL is equal to that of PCL blocks in PCL- $b$-PB though there is a large difference in the crystallization rate between neat PCL and PCL blocks. When $\xi>500 \mathrm{~nm}$, on the other hand, the crystallization rate of PCL deviates significantly from that of PCL blocks, and they approach to the values of neat polymers with increasing $\xi$. The relation between the crystallization rate and the pre-existing composition heterogeneity is quantitatively discussed. [DOI 10.1295/polymj.37.584]

KEY WORDS Crystallization / Phase Separation / Crystalline-Amorphous Diblock Copolymer / Binary Blend / Small-Angle X-Ray Scattering / Synchrotron Radiation /
\end{abstract}

It is well known that crystallization is an important factor for morphology formation in polymer blend systems. Many experimental studies on the crystallization behavior and resulting morphology were performed by using completely miscible homopolymer blends, ${ }^{1-3}$ because it is easier to understand the crystallization mechanism of such blends in which other factors for morphology formation, such as phase separation between components and microphase separation of block copolymers, are completely excluded. When a binary blend has an UCST- or LCST-type phase separation in a same temperature region of crystallization of constituent polymers, we have to consider a cooperative effect between crystallization and phase separation for the morphology formation, which will be more complicated to understand than the case of miscible blends. However, the interplay between phase separation and crystallization will be more important for the practical purpose, so that many studies have been reported so far on this subject by using binary blends of crystalline/amorphous homopolymers. ${ }^{4-19}$ However, a limited number of studies is available on this subject for binary blends of crystalline/crystalline polymers, ${ }^{20-23}$ though it might be possible to get more information on the morphology formation because two kinds of crystallization process will be detected.

We have previously investigated the morphology formation in a binary blend of poly( $\varepsilon$-caprolactone) (PCL) and polystyrene, ${ }^{4,8,9}$ where an UCST-type phase separation took place at the same temperature range of the PCL crystallization. We used the homogeneous off-critical blends, that is, the phase separation was not experienced before crystallization, to clarify the effect of demixing region existing in the phase diagram on the subsequent crystallization and resulting morphology. In addition, we recently studied the morphology formation in a double crystalline blend of PCL and PCL-block-polybutadiene (PCL- $b$ $\mathrm{PB}),{ }^{23}$ where the blend was quenched from a microphase-separated melt into various crystallization temperatures of PCL chains (i.e., PCL and PCL blocks in PCL- $b$-PB) without passing through the UCST-type demixing region. We have found from these studies that the overall morphology formation is driven by the crystallization of constituent polymers but the details of resulting morphology are controlled by the cooperative effect of crystallization and phase separation even though the macroscopic phase separation does not take place before crystallization. In particular, in the PCL/PCL- $b$-PB blend, the crystallization rate of PCL is equal to that of PCL blocks, which implies that crystallized PCL regions always coexist with crystallized PCL- $b$-PB regions and the volume ratio of these two regions is constant throughout the morphology formation.

'To whom correspondence should be addressed (TEL: +81-3-5734-2132, Fax: +81-3-5734-2888, E-mail: snojima@polymer.titech.ac.jp). 
In this study, we investigate the effect of heterogeneous structure formed by the phase separation on the subsequent crystallization behavior of PCL chains in the same binary blend of PCL and PCL- $b$-PB. That is, we focus our attention on the characteristic length of phase-separated structure existing prior to the crystallization. The crystallization process of this system is again pursued by time-resolved small-angle X-ray scattering with synchrotron radiation (SR-SAXS). From these results, we aim to clarify the relationship between the characteristic length of pre-existing phase-separated structure and the crystallization rate of PCL and PCL blocks, and discuss the crystallization mechanism in this system.

\section{EXPERIMENTAL}

\section{Polymers and Sample Preparation}

PCL $\left(M_{\mathrm{n}}=3,500\right.$ and $\left.M_{\mathrm{w}} / M_{\mathrm{n}}=1.50\right)$ and PCL- $b$ PB $\left(M_{\mathrm{n}}=13,000, M_{\mathrm{w}} / M_{\mathrm{n}}=1.16\right.$, and PCL:PB $=$ 66:34 in volume ratio) used in this study are the same that we used in our previous study. ${ }^{23}$ This blend has an UCST-type phase separation with the critical temperature at $c a .110^{\circ} \mathrm{C}$, and the melting temperatures of PCL chains (i.e., PCL and PCL blocks in PCL- $b$ PB) are $c a .60^{\circ} \mathrm{C}$ irrespective of composition. The melting temperatures meet with the cloud point curve at $\phi_{\mathrm{PCL}} \sim 0.2$ and 0.8 , where $\phi_{\mathrm{PCL}}$ is the weight fraction of PCL in the blend, so that the blends with $0.2<$ $\phi_{\mathrm{PCL}}<0.8$ take a macroscopic phase separation before the crystallization of PCL chains when they are quenched from a microphase-separated melt into various crystallization temperatures $T_{\mathrm{c}}$.

The thermal history applied to the sample is shown in Figure 1. The microphase-separated blends with $0.2<\phi_{\mathrm{PCL}}<0.8$ at $120^{\circ} \mathrm{C}$ were quenched into $70^{\circ} \mathrm{C}\left(>T_{\mathrm{m}}\right)$ and annealed there for a prescribed time $t_{\mathrm{p}}$, by which the phase separation between PCL and

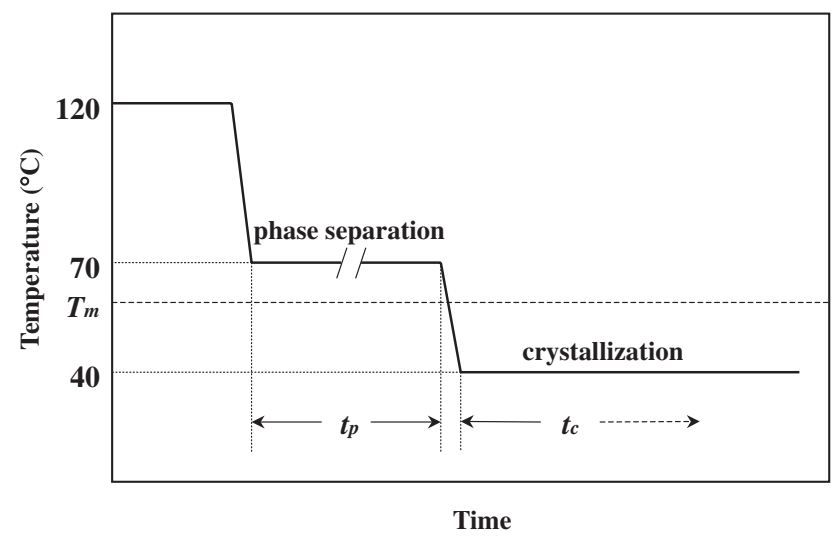

Figure 1. Thermal history applied to the sample. The sample was annealed at $70^{\circ} \mathrm{C}$ for $t_{\mathrm{p}}\left(0 \mathrm{~h}<t_{\mathrm{p}}<42 \mathrm{~h}\right)$ to get various phase-separated structures.
PCL- $b$-PB took place to result in a heterogeneous structure, that is, one was rich in PCL and the other rich in PCL- $b$-PB. The characteristic length of this heterogeneous structure could be controlled by changing $t_{\mathrm{p}}$ at $70^{\circ} \mathrm{C}$. Finally the blend was quenched into $T_{\mathrm{c}}$ $\left(=40^{\circ} \mathrm{C}\right.$ in this study) and from this moment the crystallization process of PCL chains was pursued by SR-SAXS method. The phase separation at $T_{\mathrm{c}}$ is extremely slow, so that further phase separation can be neglected during the crystallization of PCL chains.

\section{Transmission Electron Microscopy (TEM) Observa- tions}

The morphology of phase-separated blends was observed by TEM (JEOL model 200CX with an acceleration voltage of $120 \mathrm{kV})$. The phase-separated samples with various $t_{\mathrm{p}}$ were exposed to $\mathrm{RuO}_{4}$ vapor at room temperature for $15 \mathrm{~min}$ to give the contrast and also to fix the morphology, and then microtomed at room temperature into a sliver with a $c a .55 \mathrm{~nm}$ in thickness. $\mathrm{RuO}_{4}$ reacted with the double bond of polybutadiene blocks to present dark contrast.

The TEM pictures were quantitatively analyzed to evaluate the characteristic length of composition variation $\xi$ caused by the phase separation between PCL and PCL- $b$-PB. First we divided the TEM picture into two regions, one was rich in PCL and the other rich in PCL$b$-PB, with the help of contrast difference between stained and unstained regions by $\mathrm{RuO}_{4}$ and the characteristic morphology for the crystallized PCL- $b$-PB (enclosed, for example, by circles in Figure 2). This picture showed an interconnected periodic structure usually observed at the late stage of spinodal decomposition in polymer blends with a critical composition, ${ }^{24,25}$ though the characteristic length in our system was fairly small. The value of $\xi$ was finally evaluated from the statistical average of the periodic length of this structure. This method for evaluating $\xi$ might accompany a moderate error, but it is small enough when compared with the variation of $\xi$ (Figure 6) and therefore does not change our conclusion at all.

\section{SR-SAXS Measurements}

The SR-SAXS measurement was performed at Photon Factory in High-Energy Accelerator Research Organization, Tsukuba Japan, with a small-angle $\mathrm{X}$-ray equipment for solution (SAXES) installed at beam line BL-10C. Details of the optics and the instrumentation were described elsewhere. ${ }^{26,27}$ The SR-SAXS measurements were continued until the integrated intensity did not change any more, and each time-sliced SAXS intensity during $10 \mathrm{~s}$ was obtained as a function of wave number $s\left(=\frac{2}{\lambda} \sin \theta ; 2 \theta\right.$ : scattering angle, $\lambda$ : X-ray wave length $(=0.1488 \mathrm{~nm}))$ after correcting background scattering. In this study, we 

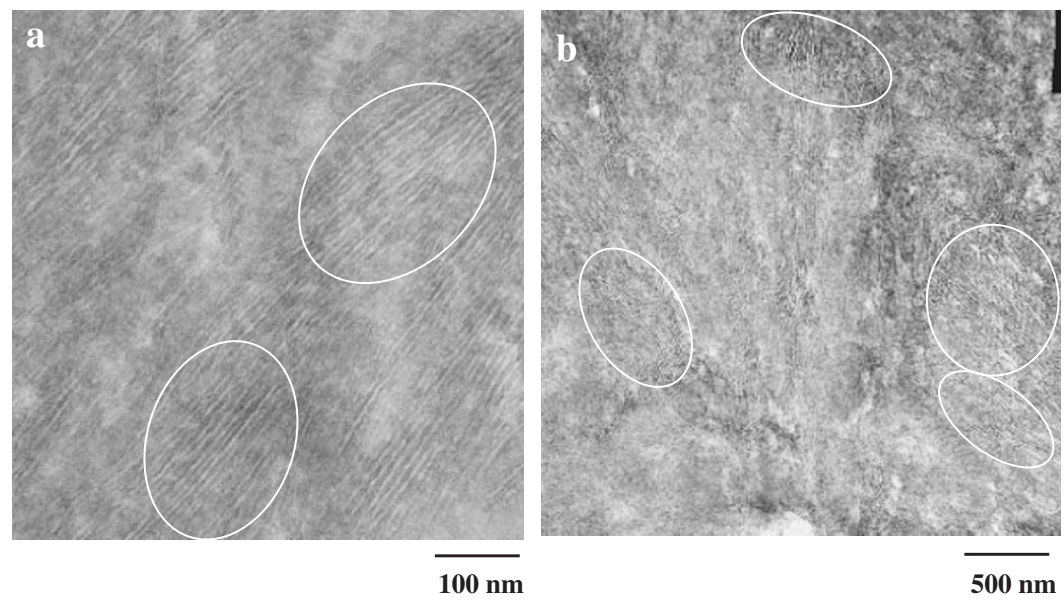

Figure 2. TEM pictures of phase-separated blends with $\phi_{\mathrm{PCL}}=0.6$ annealed at $70^{\circ} \mathrm{C}$ for $3 \mathrm{~h}$ (a) and $24 \mathrm{~h}$ (b). Typical crystallized PCL$b$-PB regions are enclosed by circles for qualitative understanding of the phase-separated structure. Note that the magnification is different between (a) and (b).

focused our attention on the time dependence of the scattered peak intensity from the lamellar morphology, i.e, an alternating structure consisting of thin crystals and amorphous layers, because it is straightforwardly obtained from the experimental results without further calculation.

\section{RESULTS}

\section{Development of Phase-Separated Structure}

Figure 2 shows typical TEM pictures for the blend with $\phi_{\mathrm{PCL}}=0.6$, which was macroscopically phaseseparated at $70^{\circ} \mathrm{C}$ for $3 \mathrm{~h}$ (a) and $24 \mathrm{~h}$ (b). It reflects the phase-separated structure just before the crystallization of PCL chains because the crystallization rate of PCL chains at room temperature is extremely large and eventually PCL chains crystallize instantaneously to prevent further macroscopic phase separation. This technique for fixing the macroscopically phase-separated structure by the subsequent crystallization is sometimes used for the morphological control in polymer blends. ${ }^{5}$ We can see two regions in these TEM pictures; (1) dark region stained by $\mathrm{RuO}_{4}$ in which regular alternating structures consisting of white and black strips are scattered (typical areas are indicated by circles) and (2) relatively bright region with no definite structure, which contains few PB blocks and therefore mainly consists of PCL homopolymers. As a result, the TEM picture divided into two regions showed an interconnected periodic structure usually observed at the late stage of spinodal decomposition in critical polymer blends. ${ }^{24,25}$

We evaluated from Figure 2 the characteristic length of composition variation $\xi$ on the basis of Figure 2 and plotted in Figure 3 against phase separation time $t_{\mathrm{p}}$. The time evolution of $\xi$ shows a linear relation in a double logarithmic plot, indicating that

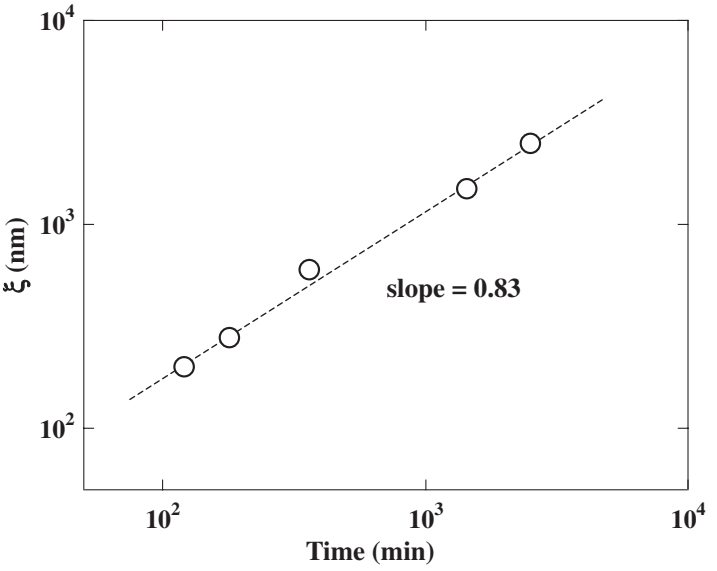

Figure 3. The characteristic length of composition variation $\xi$ plotted against phase separation time $t_{\mathrm{p}}$ for the blend with $\phi_{\mathrm{PCL}}=0.6$, which was phase-separated at $70^{\circ} \mathrm{C}$.

a power-law relation, $\xi \propto t_{\mathrm{p}}{ }^{\alpha}$, holds between $\xi$ and $t_{\mathrm{p}}$. The exponent $\alpha$ in this case is 0.83 , which is slightly smaller than the predicted value $(\alpha=1)^{28}$ and coincident with the experimental value $(\alpha=0.8)$ for the late stage of spinodal decomposition in a critical blend of polystyrene and poly(vinyl methyl ether). ${ }^{29}$ It is found from Figure 3 that we can control $\xi$ from 200 to $2500 \mathrm{~nm}$ by changing $t_{\mathrm{p}}$ at $70^{\circ} \mathrm{C}$, and therefore we can investigate the effect of $\xi$ on the subsequent crystallization behavior of PCL and PCL- $b$-PB.

\section{Overview of Crystallization Process}

Figure 4 shows typical time-resolved SAXS curves during the crystallization at $40^{\circ} \mathrm{C}$ for the blend with $\phi_{\mathrm{PCL}}=0.6$ directly quenched from the microphaseseparated melt at $120^{\circ} \mathrm{C}$ (a) and annealed first at $70^{\circ} \mathrm{C}$ for $42 \mathrm{~h}$ for phase separation prior to the crystallization (b). The SAXS peak arising from the microdomain structure (indicated by A) gradually decreases 


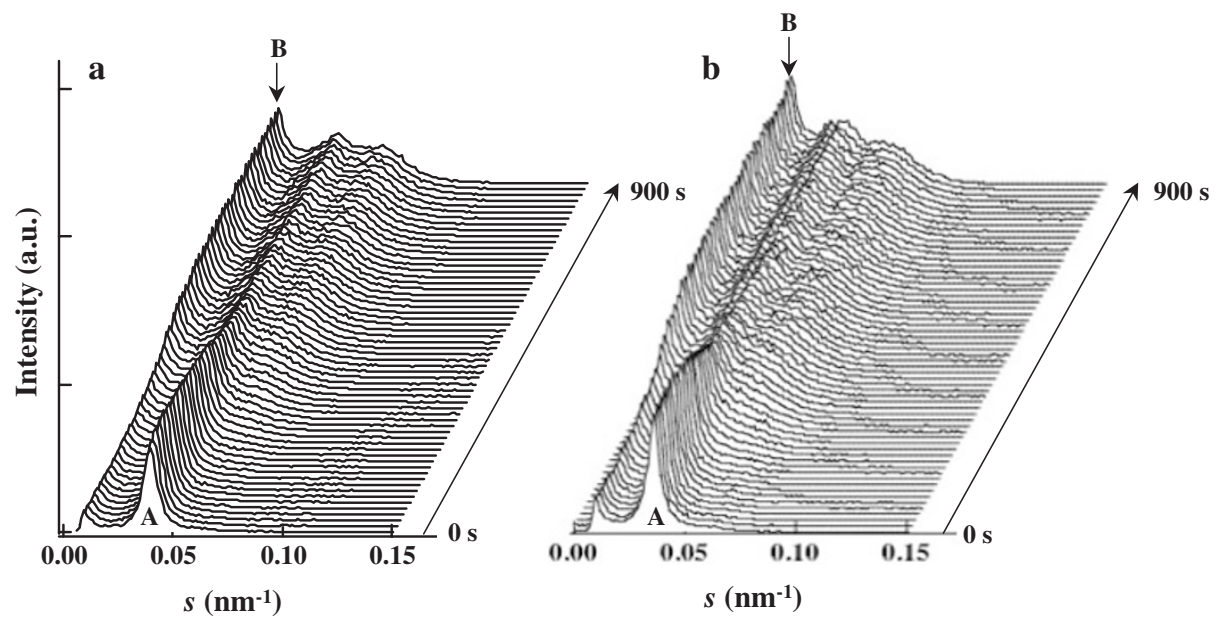

Figure 4. SR-SAXS curves during crystallization for the blend with $\phi_{\mathrm{PCL}}=0.6$ quenched from $120^{\circ} \mathrm{C}$ into $40^{\circ} \mathrm{C}$ (a) and phaseseparated at $70^{\circ} \mathrm{C}$ for $42 \mathrm{~h}$ prior to the crystallization at $40^{\circ} \mathrm{C}(\mathrm{b})$. The SAXS peak arising from the microdomain structure (indicated by A) is replaced by two scattered peaks at lower and higher angles, which come from the crystallized PCL- $b$-PB regions and PCL regions, respectively. In addition, a strong scattering toward $s \rightarrow 0$ appears (indicated by B), which arises from the mosaic structure consisting of the crystallized PCL and PCL- $b$-PB regions.

in intensity, and finally it is replaced by two scattered peaks at higher and lower angles, which arise from the crystallized PCL region and PCL- $b$-PB region, respectively, judging from a large difference in spacing; the spacing of lamellar morphology formed in crystalline block copolymers is generally larger than that of homopolymers because the amorphous block (PB block) inevitably intervenes between lamellar crystals. ${ }^{27}$ In addition, a strong scattering toward $s \rightarrow 0$ appears (indicated by B) and it's intensity increases with increasing crystallization time $t_{\mathrm{c}}$. This scattering was ascribed to the mosaic structure consisting of PCL and PCL- $b$-PB regions in our previous studies. ${ }^{8,9,23}$ If we compare two SR-SAXS curves in detail, we can notice an important difference between them. The SAXS peak at higher angle (arising from PCL regions) appears at short time in (b) compared with the case of (a), indicating that the crystallization of PCL proceeds faster in the phase-separated blend than that in the quenched blend.

The normalized peak intensity, i.e., peak intensity divided by the final intensity, for the PCL and PCL$b$-PB regions is plotted against $t_{\mathrm{c}}$ in Figure 5 for the blend with $\phi_{\mathrm{PCL}}=0.6$ quenched from the melt (a) and annealed at $70^{\circ} \mathrm{C}$ for $42 \mathrm{~h}$ prior to the crystallization (b). The peak intensity arising from both the regions in the quenched blend increases in a same fashion with increasing $t_{\mathrm{c}}$, as found in our previous study, ${ }^{23}$ suggesting that the crystallization rate of both regions is identical though the crystallization rate of neat PCL is much larger than that of neat PCL- $b$ $\mathrm{PB}$, and therefore two regions grow simultaneously with $t_{\mathrm{c}}$ to yield a constant volume ratio throughout morphology formation. In the annealed blend, on the
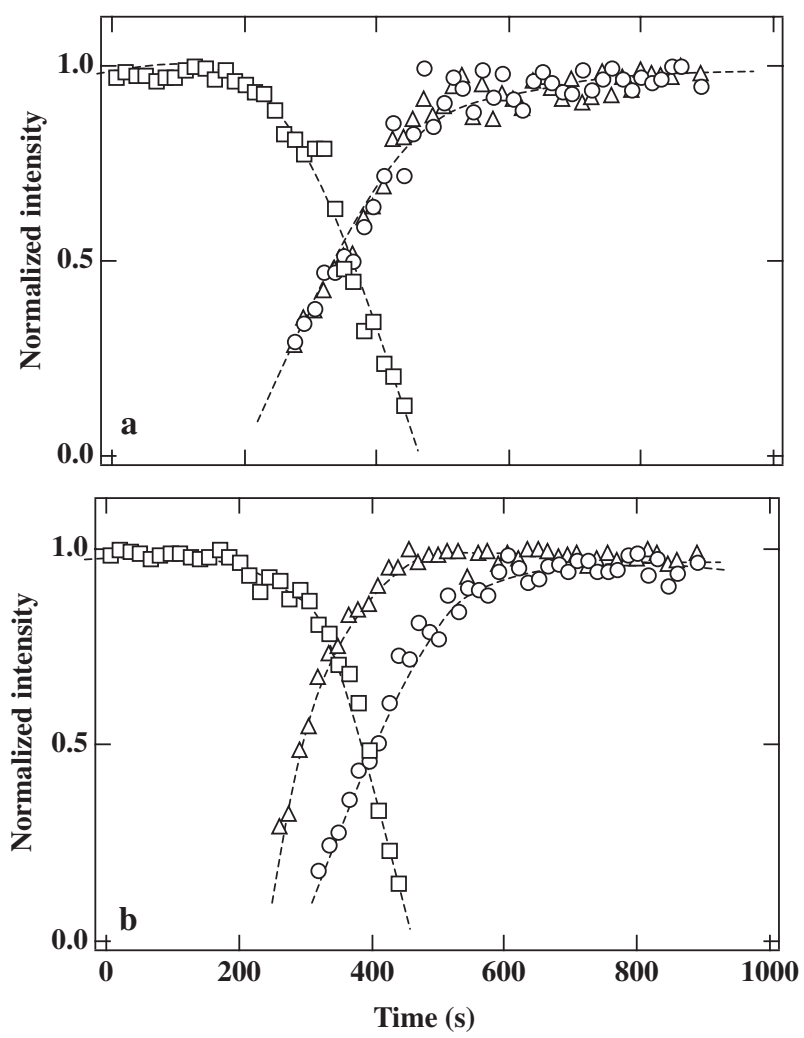

Figure 5. Normalized peak intensities plotted against crystallization time $t_{\mathrm{c}}$ for the blend with $\phi_{\mathrm{PCL}}=0.6$ quenched from $120^{\circ} \mathrm{C}$ into $40^{\circ} \mathrm{C}$ (a) and phase-separated at $70^{\circ} \mathrm{C}$ for $42 \mathrm{~h}$ prior to the crystallization at $40^{\circ} \mathrm{C}$ (b). The symbols stand for the peak intensity from the microdomain structure $(\square)$, PCL region $(\triangle)$, and PCL- $b$-PB region $(\bigcirc)$.

other hand, the increase of peak intensity for the PCL region is faster than that for the PCL- $b$-PB region, as shown in Figure $5 \mathrm{~b}$. This fact comes intuitively by 
considering that each region is enough large to yield the independent crystallization within it, and leads the important conclusion that the volume ratio of two regions is no longer constant throughout the morphology formation. It is therefore necessary to quantitatively analyze the crystallization behavior of annealed blends to clarify this point, which will be discussed later.

\section{Relation between Crystallization Rate and Character- istic Length of Composition Variation}

The half-time of crystallization $t_{1 / 2}$, which is a measure of crystallization rate, can be evaluated from the $t_{\mathrm{c}}$ dependence of each SAXS peak (Figure 5), and is plotted in Figure 6 against $\xi$ obtained from Figure 3. When $\xi$ is less than $500 \mathrm{~nm}$ the crystallization rate of PCL and PCL- $b$-PB regions is identical and intermediate between those of neat polymers (indicated by closed symbols in Figure 6). However, the difference becomes significant when $\xi$ goes beyond $500 \mathrm{~nm}$, and they approach to the crystallization rate of neat polymers. This is the new finding of this study and clearly shows that the crystallization rate is intimately dependent on the characteristic length of composition variation existing in the system.

The crystallization has quantitatively been investigated so far for some binary blends consisting of crystalline/amorphous homopolymers as a function of phase separation time $t_{\mathrm{p} .}{ }^{14,16,18}$ Lee et al. ${ }^{16}$ for example, investigated the crystallization behavior of a binary blend of poly(ethylene terephthalate) (PET) and poly(ether imide), and found that the crystallization rate of PET decreased significantly with increasing $t_{\mathrm{p}}$, which means that the phase-separated structure is disadvantageous to the crystallization of PET. Kim et al. ${ }^{14}$ investigated the $t_{\mathrm{p}}$ dependence of crystallization rate for a binary blend of poly(ethylene-ran-vinyl

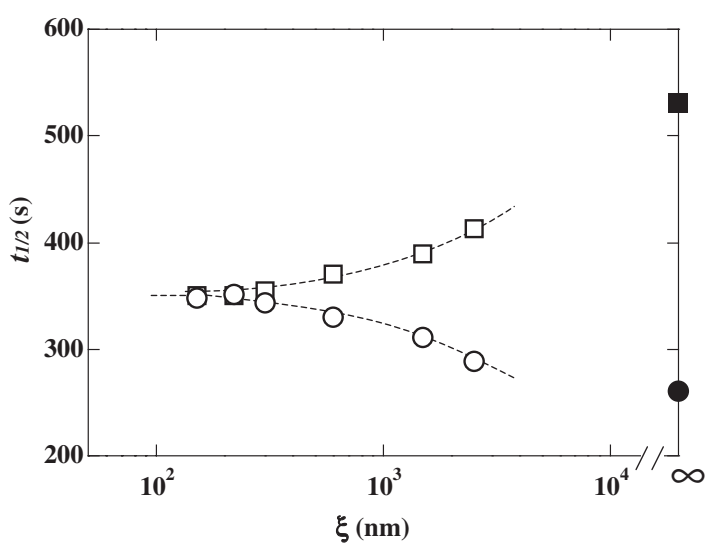

Figure 6. Half-time of crystallization $t_{1 / 2}$ for PCL $(\bigcirc)$ and PCL- $b$-PB $(\square)$ regions plotted against $\xi$ for the blend with $\phi_{\mathrm{PCL}}=0.6$. The closed symbols stand for $t_{1 / 2}$ of neat PCL

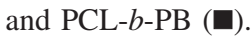

acetate) and paraffin with an UCST-type phase diagram and obtained a completely reverse result; the crystallization rate increased with increasing $t_{\mathrm{p}}$.

These experimental results all suggest that the phase separation between components certainly affects the subsequent crystallization, and also that details of influence depend intimately on the characteristics of binary blends investigated. In fact, our system shows two opposite crystallization behaviors; the crystallization of PCL- $b$-PB is accelerated while that of PCL decelerated by the phase separation. Therefore, in order to understand the effect of phase separation on the subsequent crystallization behavior and final morphology we have to consider how the factors controlling the crystallization behavior, such as viscosity (or mobility of crystalline polymers), nucleus density, or morphological restriction, change by the phase separation occurring prior to the crystallization.

\section{Crystallization Mechanism of Each Region}

Here we investigate qualitatively the crystallization mechanism of PCL and PCL- $b$-PB regions, which have different crystallization rates for the phase-separated blend, as shown in Figure 5b. In our previous studies, ${ }^{9,23}$ the crystallization mechanism was evaluated by plotting the normalized peak intensity against reduced time $\left(=t_{\mathrm{c}} / t_{1 / 2}\right)$; if two curves are identical throughout the crystallization process, the crystallization mechanism is considered to be the same.

Figure 7 shows the normalized peak intensity from PCL and PCL- $b$-PB regions plotted against reduced time for the quenched blend $(\triangle, \nabla)$ and phase-separated blend at $70^{\circ} \mathrm{C}$ for $42 \mathrm{~h}(\bigcirc, \square)$. Although the $t_{\mathrm{c}}$ dependence of normalized peak intensity shows different curves in Figure 5b, they make one master curve in Figure 7 irrespective of thermal history applied to the blend, suggesting that the crystallization

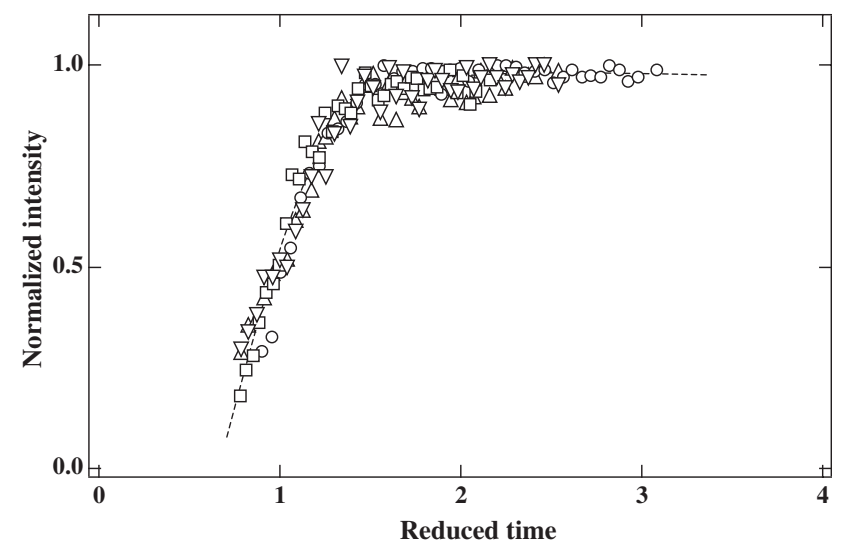

Figure 7. Normalized intensity for PCL $(\triangle)$ and PCL- $b$-PB $(\nabla)$ regions in the quenched blend and PCL $(\bigcirc)$ and PCL- $b$-PB $(\square)$ regions in the phase-separated blend plotted against reduced time $\left(=t_{\mathrm{c}} / t_{1 / 2}\right)$ for the blend with $\phi_{\mathrm{PCL}}=0.6$ at $70^{\circ} \mathrm{C}$ for $42 \mathrm{~h}$. 
mechanism of each region for the quenched and phase-separated blends is the same, and probably equivalent to that of neat PCL and PCL- $b$-PB. Therefore we can conclude that any specific crystallization mechanism does not appear in the macroscopically phase-separated blend.

\section{DISCUSSION}

Development of Crystallized PCL and PCL-b-PB Regions

In our previous study, ${ }^{23}$ we introduced the volume fraction of crystallized PCL- $b$-PB regions against total crystallized regions at $t_{\mathrm{c}}, \theta\left(t_{\mathrm{c}}\right)$, defined as,

$$
\theta\left(t_{\mathrm{c}}\right)=\frac{V_{\mathrm{PCL}-b-\mathrm{PB}}\left(t_{\mathrm{c}}\right)}{V_{\mathrm{PCL}-b-\mathrm{PB}}\left(t_{\mathrm{c}}\right)+V_{\mathrm{PCL}}\left(t_{\mathrm{c}}\right)}
$$

where $V_{\mathrm{PCL}-b-\mathrm{PB}}\left(t_{\mathrm{c}}\right)$ and $V_{\mathrm{PCL}}\left(t_{\mathrm{c}}\right)$ are the volume of the crystallized PCL- $b$-PB and PCL regions, respectively. By assuming that $V_{\mathrm{PCL}-b-\mathrm{PB}}\left(t_{\mathrm{c}}\right)$ and $V_{\mathrm{PCL}}\left(t_{\mathrm{c}}\right)$ are proportional to the normalized peak intensity from these regions, equation (1) is rewritten as,

$$
\theta\left(t_{\mathrm{c}}\right) \propto \frac{\tilde{I}_{\mathrm{PCL}-b-\mathrm{PB}}\left(t_{\mathrm{c}}\right)}{\tilde{I}_{\mathrm{PCL}-b-\mathrm{PB}}\left(t_{\mathrm{c}}\right)+\frac{\phi_{\mathrm{PCL}}^{\prime}}{1-\phi_{\text {PCL }}^{\prime}} \tilde{I}_{\mathrm{PCL}}\left(t_{\mathrm{c}}\right)}
$$

where $\tilde{I}_{\mathrm{PCL}}\left(t_{\mathrm{c}}\right)$ and $\tilde{I}_{\mathrm{PCL}-b-\mathrm{PB}}\left(t_{\mathrm{c}}\right)$ are the normalized peak intensity from the crystallized PCL-rich and PCL- $b$-PB-rich regions, respectively, and $\phi_{\mathrm{PCL}}^{\prime}$ is the volume fraction of PCL in the blend. In our previous study, where the crystallization starts from a microphase-separated state without passing through the macroscopically phase-separated region, the $t_{\mathrm{c}}$ dependence of $\tilde{I}_{\text {PCL-b-PB }}\left(t_{\mathrm{c}}\right)$ is identical with that of $\tilde{I}_{\mathrm{PCL}}\left(t_{\mathrm{c}}\right)$, so that $\theta\left(t_{\mathrm{c}}\right)$ is simply reduced to $1-\phi_{\mathrm{PCL}}^{\prime}$ $\left(\approx 1-\phi_{\mathrm{PCL}}\right)$. This means that $\theta\left(t_{\mathrm{c}}\right)$ is independent of $t_{\mathrm{c}}$ during crystallization and absolutely determined by the initial blend composition.

In the present study, however, $\tilde{I}_{\mathrm{PCL}}\left(t_{\mathrm{c}}\right)$ is significantly different from $\tilde{I}_{\mathrm{PCL}-b \text {-PB }}\left(t_{\mathrm{c}}\right)$ at large $\xi$, as shown in Figure $5 \mathrm{~b}$, and consequently $\theta\left(t_{\mathrm{c}}\right)$ is intimately dependent on $t_{\mathrm{c}}$. It is possible to evaluate the ratio $\tilde{I}_{\mathrm{PCL}-b-\mathrm{PB}}\left(t_{\mathrm{c}}\right) / \tilde{I}_{\mathrm{PCL}}\left(t_{\mathrm{c}}\right)$ from Figure $5 \mathrm{~b}$, and we can calculate the $t_{\mathrm{c}}$ dependence of $\theta\left(t_{\mathrm{c}}\right)$, which is plotted in Figure 8 for various $t_{\mathrm{p}}$ (or $\xi$ ) investigated.

As expected from the previous argument, $\theta\left(t_{\mathrm{c}}\right)$ is constant irrespective of $t_{\mathrm{c}}$ at small $\xi$, indicating that two crystallized regions, PCL and PCL- $b$-PB regions, grow simultaneously with increasing $t_{\mathrm{c}}$. At large $\xi$, on the other hand, $\theta\left(t_{\mathrm{c}}\right)$ is small at first and increases with increasing $t_{\mathrm{c}}$ to approach to the final value. This means that at the beginning of crystallization, the crystallized PCL regions grow first in the system followed by the crystallization of PCL- $b$-PB regions,

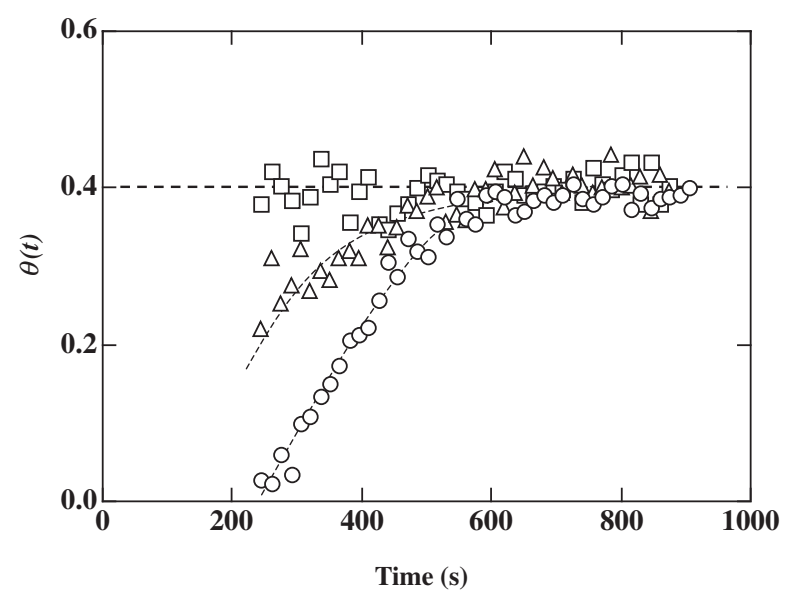

Figure 8. Volume fraction of PCL- $b$-PB regions against total crystallized regions $\theta\left(t_{\mathrm{c}}\right)$ plotted against $t_{\mathrm{c}}$ for the phase-separated blend for $3 \mathrm{~h}(\square), 6 \mathrm{~h}(\triangle)$, and $42 \mathrm{~h}(\bigcirc)$. Other plots are omitted for legibility.

which directly reflects the difference in half-time of crystallization between PCL and PCL- $b-\mathrm{PB}$, as depicted in Figure 6.

Figure 8 clearly shows that the crystallization behavior of PCL and PCL blocks in our blend depends significantly on the characteristic length of composition variation caused by the phase separation between PCL and PCL- $b$-PB. It is also important what factors influence the limited value of $\xi$ at which the difference in crystallization rate becomes significant.

\section{Limit of $\xi$ for Simultaneous Crystallization}

Here, we qualitatively discuss the limited value of $\xi$, $\xi_{0}$, for the simultaneous crystallization of PCL and PCL blocks. The phase separation time at $70^{\circ} \mathrm{C}$ is enough longer ( $3 \mathrm{~h}<t_{\mathrm{p}}<42 \mathrm{~h}$ ), so that each phaseseparated region has final compositions determined by the phase diagram of this system. Therefore, the crystallization rate of each region does not depend on the dynamics of phase separation, as pointed out for the binary blend of polycarbonate and poly(ethylene oxide). ${ }^{17}$ Here, it is necessary to consider the nucleation rate $I$ and the crystal growth rate $G$ for PCL and PCL blocks to understand $\xi_{0}$, because it is well known that the overall crystallization rate is determined by these two factors. Both rates are described by the sum of free energy associated with the activation energy for diffusion and that necessary to create the stable nucleus. If we assume that the nucleation occurs heterogeneously at the interface between phase-separated PCL-rich and PCL- $b$-PB-rich regions, $I$ for PCL will be the same to that for PCL blocks, so that what we have to consider is the relation between $G$ for PCL and PCL blocks and the size of each region.

When $\xi$ is enough small, the crystal growth will finish instantaneously after nucleation, which is 
reminiscent of the crystallization in confined spherical microdomains formed in crystalline-amorphous diblock copolymers, ${ }^{30,31}$ where the crystal growth progresses only over several tens $\mathrm{nm}$. Therefore, even if the growth rate of each region is different it does not matter and the nucleation rate drives the overall crystallization. When $\xi$ is extremely large, on the other hand, the crystal growth rate drives substantial crystallization and eventually the difference in overall crystallization rate between neat PCL and PCL blocks becomes significant in this case.

We can change the above relation between $G$ and $\xi$ by changing the crystallization temperature, because $G$ is critically dependent on the difference between the equilibrium melting temperature and crystallization temperature $T c$. We are now studying the $T c$ dependence of crystallization rates in this system, and the results will soon appear.

\section{CONCLUSIONS}

We have investigated the crystallization behavior of PCL chains (i.e., PCL homopolymer and PCL blocks in PCL- $b$-PB) in a binary blend of PCL and PCL- $b$-PB as a function of the characteristic length of composition variation $\xi$ formed in advance by the macroscopic phase separation by time-resolved small-angle X-ray scattering with synchrotron radiation. This system has an UCST-type phase-separated region in the temperature range of crystallization of PCL chains, so that the blends with $0.2<\phi_{\mathrm{PCL}}<0.8$ take the macroscopic phase separation to yield various $\xi$ prior to the crystallization. Following conclusions were obtained from the present results.

1) When $\xi<500 \mathrm{~nm}$, the crystallization rate of PCL is equal to that of PCL blocks, and consequently the crystallized PCL region grows simultaneously with the crystallized PCL- $b$-PB region.

2) When $\xi>500 \mathrm{~nm}$, the crystallization rate of PCL deviates significantly from that of PCL blocks and eventually they approach to the values of neat polymers with increasing $\xi$. This means that the crystallized PCL regions grow first followed by the crystallization of PCL- $b$ $\mathrm{PB}$ regions in the system.

Above facts clearly indicate that the characteristic length of composition variation intimately influences the crystallization behavior, for which the relation between $\xi$ and the crystal growth rate $G$ is important to understand the crystallization mechanism in this system.

Acknowledgment. This study was partially supported by NEDO (New Energy and Industrial Technology Development Organization) launched in 2001.
The SR-SAXS measurement has been performed under the approval of Photon Factory Advisory Committee (No. 2004G093). We appreciate Mr. J. Koki, Center for Advanced Materials Analysis, Tokyo Institute of Technology, for TEM observations.

\section{REFERENCES}

1. D. R. Paul and S. Newman, Ed., "Polymer Blends," Vol. 1, Academic Press, New York, N.Y., 1978.

2. R. S. Stein, F. B. Khambatta, F. P. Warner, T. P. Russell, A. Escala, and E. Balizer, J. Polym. Sci., Polym. Symp., 63, 313 (1978).

3. L. A. Utracki, "Polymer Alloys and Blends," Hanser Publisher, Munich, 1989.

4. S. Nojima, Y. Terashima, and T. Ashida, Polymer, 27, 1007 (1986).

5. N. Inaba, K. Sato, S. Suzuki, and T. Hashimoto, Macromolecules, 19, 1690 (1986).

6. W. R. Burghardt, Macromolecules, 22, 2482 (1989).

7. H. Tanaka and T. Nishi, Phys. Rev. A, 39, 783 (1989).

8. S. Nojima, K. Satoh, and T. Ashida, Macromolecules, 24, 942 (1991).

9. S. Nojima, K. Kato, M. Ono, and T. Ashida, Macromolecules, 25, 1922 (1992).

10. N. Kinami, T. Okuyama, M. Okamoto, and T. Inoue, Polymer, 36, 4449 (1995).

11. H. L. Chen and M. S. Hsiao, Macromolecules, 31, 6579 (1998).

12. H. L. Chen, J. C. Hwang, J. M. Yang, and R. C. Wang, Polymer, 39, 6983 (1998).

13. H. M. Shabana, R. H. Olley, D. C. Bassett, and B. J. Jungnickel, Polymer, 41, 5513 (2000).

14. J. K. Kim, B. K. Kim, and M. Park, J. Polym. Sci., B, 38, 707 (2000).

15. H. J. Bang, J. K. Lee, and K. H. Lee, J. Polym. Sci., B, 38, 2625 (2000).

16. J. K. Lee, W. S. Choi, Y. K. Kwon, and K. H. Lee, Polymer, 43, 2827 (2002).

17. M. Tsuburaya and H. Saito, Polymer, 45, 1027 (2004).

18. G. Matsuba, K. Shimizu, H. Wang, Z. Wang, and C. C. Han, Polymer, 45, 5137 (2004).

19. K. Shimizu, H. Wang, Z. Wang, G. Matsuba, H. Kim, and C. C. Han, Polymer, 45, 7061 (2004).

20. Q. Fu, Q. Zhang, Y. Peng, and R. Du, Polym. Int., 49, 539 (2000).

21. Y. Hu, Y. S. Hu, V. Topolkaraev, A. Hiltner, and E. Baer, Polymer, 44, 5681 (2003).

22. N. Yoshie, A. Asaka, and Y. Inoue, Macromolecules, 37, 3770 (2004).

23. M. Akaba and S. Nojima, Polym. J., 37, 464 (2005).

24. H. Yang, M. Shibayama, R. S. Stein, N. Shimizu, and T. Hashimoto, Macromolecules, 19, 1667 (1986).

25. A. Nakai, T. Shiwaku, H. Hasegawa, and T. Hashimoto, Macromolecules, 19, 3008 (1986).

26. T. Ueki, H. Hiragi, M. Kataoka, Y. Inoko, Y. Amemiya, Y. Izumi, H. Tagawa, and Y. Muroga, Biophys. Chem., 23, 115 (1985).

27. S. Nojima, K. Kato, S. Yamamoto, and T. Ashida, 
Macromolecules, 25, 2237 (1992).

28. E. D. Siggia, Phys. Rev., A, 20, 595 (1979).

29. T. Hashimoto, M. Itakura, and B. Shimizu, J. Chem. Phys., 85, 6773 (1986).
30. Y. L. Loo and R. A. Register, Phys. Rev. Lett., 84, 4120 (2000).

31. S. Nojima, M. Toei, S. Hara, S. Tanimoto, and S. Sasaki, Polymer, 43, 4087 (2002). 